\title{
A holding cost bound for the economic lot-sizing problem with time-invariant cost parameters
}

\author{
Wilco van den $\mathrm{Heuvel}^{a}{ }^{*}$, Albert P.M. Wagelmans ${ }^{a}$ \\ ${ }^{a}$ Econometric Institute and Erasmus Research Institute of Management, \\ Erasmus University Rotterdam, P.O. Box 1738, 3000 DR Rotterdam, \\ The Netherlands
}

ECONOMETRIC Institute Report EI 2008-10

April 29, 2008

\begin{abstract}
In this paper we derive a new structural property for an optimal solution of the economic lot-sizing problem with time-invariant cost parameters. We show that the total holding cost in an order interval of an optimal solution is bounded from above by a quantity proportional to the setup cost and the logarithm of the number of periods in the interval. Since we can also show that this bound is tight, this is in contrast to the optimality property of the economic order quantity (EOQ) model, where setup cost and holding cost are perfectly balanced. Furthermore, we show that this property can be used for the design of a new heuristic and that the result may be useful in worst case analysis.
\end{abstract}

\section{Introduction}

In this paper we consider the well-known economic lot-sizing (ELS) model introduced in the seminal paper by Wagner and Whitin (1958). The model has a finite and discrete time horizon of $T$ periods and in each period $t=1, \ldots, T$ there is a demand $d_{t}$. The problem is to determine the order periods and order quantities such that total costs are minimized. Costs include a fixed setup cost $K$ for every order placed, and a unit holding cost $h$ per period for every item held in stock. (Note that the terms setup cost and order cost are both used in the literature dependent on the context of the problem.)

Although the ELS problem can be solved efficiently (see Federgruen and Tzur (1991), Wagelmans et al. (1992) and Aggarwal and Park (1993)), many heuristics have been proposed in the

\footnotetext{
${ }^{*}$ Corresponding author. E-mail: wvandenheuvel@few.eur.nl, Phone: +31 10 4081260, Fax: +31 104089162
} 
literature and are still used in material requirements planning (MRP) software. A number of those heuristics utilize some optimality property of the economic order quantity (EOQ) model. In contrast to the ELS model, the EOQ model assumes a constant demand rate $D$ over a continuous and infinite horizon. Having the same fixed order cost $K$ and holding cost $h$, the average cost per time unit equals

$$
\frac{K}{T}+\frac{1}{2} T h D,
$$

if an order is placed every $T$ periods. The first part represents the average order cost and the second part represents the average holding cost per time unit. The optimal time between two orders that minimizes the average cost equals $T^{*}=\sqrt{2 K /(D h)}$. It is a well-known property that the average setup and holding cost are perfectly balanced in an optimal solution, i.e., $K / T^{*}=\sqrt{K D h / 2}=\frac{1}{2} T^{*} h D$.

The Part Period Balancing (PPB) algorithm is a heuristic for the ELS model that is based on this property (see, for example, Silver et al. (1998) for a description of the heuristic). The PPB algorithm tries to construct a solution where setup cost and holding are balanced. Other heuristics also try to exploit some optimality property of the EOQ model. For example, the Silver-Meal (SM) heuristic minimizes the average cost per period, and the Least Unit Cost (LUC) heuristic minimizes the average cost per item. Wemmerlöv (1981) gives an overview of how the different rules for the ELS problem relate to the properties of an optimal solution to the EOQ model.

In this paper we are interested in the question whether the property of balancedness between setup and holding cost also holds (to some extent) for the ELS model, and hence, whether it is justified to apply heuristics based on this property. In particular, we are interested in the relation between holding cost and setup cost in an optimal solution of the ELS model. Clearly, we can have zero holding cost in case setup cost is sufficiently small (resulting in an optimal solution with an order in each period). This raises the question whether there also exist problem instances for which the total holding cost is relatively large compared to the total setup cost.

In this paper we will show that the total holding cost in an optimal order interval is bounded from above by a quantity proportional to the setup cost and the logarithm of the number of periods in the interval, where an order interval is defined as the number of consecutive periods for which demand is satisfied by a single order. This is in contrast with the optimality property of the EOQ model and so the result shows there is no justification for the rationale behind the PPB algorithm.

The remainder of the paper is organized as follows. In Section 2 we will derive the holding cost bound. In Section 3 we show how this property can be used for the design of a new heuristics. We analyze the worst case performance of the heuristic, and derive a property on the number of setups in a solution. Section 4 shows how the result can be used for worst case analysis. The paper is completed in Section 5 with the conclusion. 


\section{The main result}

It is well known that there exists an optimal solution for the ELS problem that satisfies the zero-inventory property, i.e., a new order is placed only if the inventory drops down to zero. This means that in any order period demand is ordered for an integral number of consecutive periods. Consider some order interval in an optimal solution and w.l.o.g. assume it consists of periods $1, \ldots, t$. In this section we will derive an upper bound on the total holding costs in these periods. The idea of the proof is that in an optimal solution it is never profitable to add a setup in any period $p+1$ with $p \in\{1, \ldots, t-1\}$. The total holding cost $H_{t}$ for the order interval of length $t$ equals

$$
H_{t}=h \sum_{i=2}^{t}(i-1) d_{i} .
$$

Furthermore, adding a setup in period $p+1$ leads to a saving in holding cost of

$$
p h \sum_{i=p+1}^{t} d_{i} .
$$

The following lemmas are used to derive our main theorem.

Lemma 1 Assume there exists a constant $c \geq 0$ and a period $p \in\{1, \ldots, t-1\}$ such that

$$
c p h \sum_{i=p+1}^{t} d_{i} \geq h \sum_{i=2}^{t}(i-1) d_{i} .
$$

Then in an optimal solution $H_{t} \leq c K$.

Proof Because we consider an order interval of an optimal solution, adding a setup in any period $p+1$ leads to a cost saving that is smaller than $K$. So for all $p \in\{1, \ldots, t-1\}$ we have

$$
p h \sum_{i=p+1}^{t} d_{i} \leq K .
$$

Therefore,

$$
H_{t}=h \sum_{i=2}^{t}(i-1) d_{i} \leq c p h \sum_{i=p+1}^{t} d_{i} \leq c K,
$$

where the first inequality follows from (1).

It follows from Lemma 1 that if we can find a $c$ that satisfies (1) for all demand sequences $d=d_{1}, \ldots, d_{t}$, then we have found a bound on the total holding cost in an order interval. Note that a trivial upper bound on $c$ is $t-1$. It is easy to see that (1) holds for $c=t-1$ and $p=1$, which implies that $H_{t} \leq(t-1) K$ in an optimal solution. This bound is also obtained from the fact that the cost must be smaller than the cost of the solution with a setup in every period which equals $t K$. Because the setup cost equals $K$, the holding cost must be smaller 
than $(t-1) K$. The goal is to find an upper bound $c$ that is lower than this trivial bound. In the following lemma we will give a lower bound on the value of $c$ and Lemma 3 shows that this bound is tight.

Lemma 2 Given the demand sequence $d^{0}$ defined by

$$
\begin{aligned}
d_{1}^{0} & >0, \\
d_{i}^{0} & =\frac{1}{i(i-1)}=\frac{1}{i-1}-\frac{1}{i}, \quad i=2, \ldots, t-1 \\
d_{t}^{0} & =\frac{1}{t-1} .
\end{aligned}
$$

Then for the sequence $d^{0}$ it holds

$$
\left(\sum_{i=1}^{t-1} \frac{1}{i}\right) p \sum_{i=p+1}^{t} d_{i}^{0}=\sum_{i=2}^{t}(i-1) d_{i}^{0} \text { for } p=1, \ldots, t-1 .
$$

Proof First, it holds for the right hand side that

$$
\sum_{i=2}^{t}(i-1) d_{i}^{0}=\sum_{i=2}^{t-1} \frac{1}{i}+1=\sum_{i=1}^{t-1} \frac{1}{i}
$$

Second, for any period $p \in\{1, \ldots, t-1\}$ it holds

$$
p \sum_{i=p+1}^{t} d_{i}^{0}=p\left(\sum_{i=p+1}^{t-1}\left(\frac{1}{i-1}-\frac{1}{i}\right)+\frac{1}{t-1}\right)=p\left(\left(\frac{1}{p}-\frac{1}{t-1}\right)+\frac{1}{t-1}\right)=1,
$$

which completes the proof.

Note that for the problem instance of Lemma 2, adding a setup to any period $p$ leads to the same reduction in holding cost. Because the problem instance of Lemma 2 is a specific problem instance, for an arbitrary instance we must have $c \geq \sum_{i=1}^{t-1} \frac{1}{i}$. However, the following lemma shows that the bound $\sum_{i=1}^{t-1} \frac{1}{i}$ is tight.

Lemma 3 For any demand sequence $d=d_{1}, \ldots, d_{t}$ there exists a period $p \in\{1, \ldots, t-1\}$ such that

$$
\left(\sum_{i=1}^{t-1} \frac{1}{i}\right) p \sum_{i=p+1}^{t} d_{i} \geq \sum_{i=2}^{t}(i-1) d_{i}
$$

Proof In case $d_{i}=0$ for $i=2, \ldots, t$ the lemma trivially holds. Assume that there is some period $d_{i}>0$ with $i \in\{2, \ldots, t\}$. Let $\alpha>0$ be such that

$$
\sum_{i=2}^{t}(i-1) d_{i}=\alpha \sum_{i=2}^{t}(i-1) d_{i}^{0}
$$

and define the vector

$$
\Delta d=d-\alpha d^{0}
$$


Then it holds

$$
\sum_{i=2}^{t}(i-1) \Delta d_{i}=\sum_{i=2}^{t}(i-1)\left(d_{i}-\alpha d_{i}^{0}\right)=0 .
$$

By Lemma 2 it is now sufficient to show that there exists a period $p \in\{1, \ldots, t-1\}$ such that

$$
p \sum_{i=p+1}^{t} d_{i} \geq p \sum_{i=p+1}^{t} \alpha d_{i}^{0} \Leftrightarrow p \sum_{i=p+1}^{t} \Delta d_{i} \geq 0
$$

because then

$$
\left(\sum_{i=1}^{t-1} \frac{1}{i}\right) p \sum_{i=p+1}^{t} d_{i} \geq\left(\sum_{i=1}^{t-1} \frac{1}{i}\right) p \sum_{i=p+1}^{t} \alpha d_{i}^{0}=\alpha \sum_{i=2}^{t}(i-1) d_{i}^{0}=\sum_{i=2}^{t}(i-1) d_{i} .
$$

Assume that (3) does not hold, i.e., for all $p \in\{1, \ldots, t-1\}$

$$
p \sum_{i=p+1}^{t} \Delta d_{i}<0 \Leftrightarrow \sum_{i=p+1}^{t} \Delta d_{i}<0 .
$$

But then

$$
0>\sum_{p=1}^{t-1} \sum_{i=p+1}^{t} \Delta d_{i}=\sum_{i=2}^{t} \sum_{p=1}^{i-1} \Delta d_{i}=\sum_{i=2}^{t}(i-1) \Delta d_{i}=0,
$$

which is a contradiction.

We are now ready to state our main theorem.

Theorem 4 Let periods $1, \ldots, t$ be an order interval in an optimal solution of a problem instance with demand sequence $d_{1}, \ldots, d_{t}$. Then it holds for the total holding cost

$$
H_{t}=h \sum_{i=2}^{t}(i-1) d_{i} \leq K \sum_{i=1}^{t-1} \frac{1}{i} .
$$

Proof By Lemma 3 we have that for any problem instance (2) holds. Applying Lemma 1 with $c=\sum_{i=1}^{t-1} \frac{1}{i}$ gives the result.

To derive the bound on the holding cost in an optimal order interval we used the fact that one additional setup in some period can never decrease the total cost. A result by Van Hoesel and Wagelmans (2000) shows that adding more than one setup cannot lead to a cost reduction either. Namely, Van Hoesel and Wagelmans (2000) show that the function $z(n)$ is convex, where $z(n)$ is the optimal cost of the lot-sizing problem with exactly $n$ setups.

A direct consequence of Lemma 2 is that there exists problem instances for which the ratio between holding cost and setup cost becomes arbitrarily large. Namely, for the problem instance with demand $d^{0}, K=1, h=1$ and $T$ periods an optimal solution is to have only one setup in period 1. For this instance the ratio equals $\sum_{t=1}^{T-1} \frac{1}{t} \rightarrow \infty$ as $T \rightarrow \infty$. This means that the bound is tight and so we have the following theorem. 
Theorem 5 There exist problem instances for which the ratio between holding cost and setup cost in an optimal solution is arbitrarily large.

Theorem 5 shows that the rationale behind PPB criterium is not justified because holding cost and setup cost need not be balanced in an optimal solution.

Finally, note that the sum of the first $t-1$ terms of the harmonic series $\sum_{i=1}^{t-1} \frac{1}{i}$ increases relatively slowly. It is well known that

$$
\lim _{n \rightarrow \infty}\left(\sum_{i=1}^{n} \frac{1}{i}-\log n\right)=\gamma,
$$

where $\gamma=0.577 \ldots$ is the Euler-Mascheroni constant (see e.g. Graham et al. (1994)). Using this result it is not difficult to show that

$$
\sum_{i=1}^{t-1} \frac{1}{i} \leq \gamma+\log t .
$$

So using (4) and (5) the holding cost of any optimal order interval satisfies

$$
H_{t} \leq K(\gamma+\log t)
$$

which means that the holding cost equals at most a quantity proportional to the setup cost and the logarithm of the number of periods in the order interval.

\section{A new heuristic}

Theorem 4 immediately suggests a new heuristic for the economic lot-sizing problem with timeinvariant costs. The heuristic selects an order interval that covers periods $1, \ldots, t$ with $t$ the largest period that satisfies

$$
h \sum_{i=2}^{t}(i-1) d_{t} \leq K \sum_{i=1}^{t-1} \frac{1}{i} .
$$

In this way the solution possesses a property that is satisfied by any optimal solution. We will call this heuristic $H$. Unfortunately, the following example shows that the worst case performance of $H$ can be arbitrarily bad.

Example 1 Consider a problem instance with $d_{1}>0, d_{T}=\frac{K}{h(T-1)} \sum_{t=1}^{T-1} \frac{1}{t}$ and $d_{t}=0$ for $t=2, \ldots, T-1$. Then the heuristic $H$ generates a solution with only a setup in period 1 having a total cost of $C^{H}=K+K \sum_{t=1}^{T-1} \frac{1}{t}$. However, the optimal solution is to order in periods 1 and $T$ with a total cost of $C^{*}=2 K$. Clearly, the worst case ratio equals

$$
\frac{C^{H}}{C^{*}}=\frac{K\left(1+\sum_{t=1}^{T-1} \frac{1}{t}\right)}{2 K} \rightarrow \infty \text { as } T \rightarrow \infty .
$$


In Example 1 we used $c=\sum_{i=1}^{t-1} \frac{1}{i}$, which is the smallest $c$ that satisfies (1) for an arbitrary problem instance. However, this is not the best value of $c$ given a specific problem instance. We can improve $H$ by dynamically updating $c$. This modified heuristic works as follows. Assume that we arrive in some period $t$ with the last setup in period 1 . Calculate the smallest $c$ that satisfies (1), say $c_{t}$, and check whether

$$
h \sum_{i=2}^{t}(i-1) d_{t} \leq c_{t} K .
$$

If the latter inequality holds, proceed with period $t+1$. Otherwise, make an order that covers periods $1, \ldots, t-1$, start a new order in period $t$ and proceed with period $t+1$. We will call this heuristic $H^{*}$, the 'refined' version of heuristic $H$.

Heuristic $H^{*}$ has a nice interpretation. Note that if we arrive in some period $t$ that does not satisfy (8), then there exists some period $p \in\{2, \ldots, t\}$ such that an additional setup in period $p$ leads to a cost reduction. So heuristic $H^{*}$ chooses the order intervals as large as possible (except for possibly the last order interval) such that no additional setup may improve the solution. We will use this interpretation to determine the worst case performance of $H^{*}$.

Theorem 6 The worst case performance of $H^{*}$ is at most 2.

Proof Consider a solution for some arbitrary instance $d$ generated by $H^{*}$ with $\operatorname{cost} C^{H^{*}}$. Modify this solution by adding a setup (if none yet) in every period where the optimal solution has a setup and modify the order quantities accordingly. Denote the cost of this solution by $\bar{C}$. Assume that there are $n^{*}$ setups in the optimal solution so that we add at most $n^{*}-1$ setups (both solutions have a setup in period 1) to our heuristic solution. Then we have

$$
C^{H^{*}} \leq \bar{C} \leq C^{*}+\left(n^{*}-1\right) K \leq 2 C^{*}
$$

with $C^{*}$ the cost of the optimal solution. The first inequality follows because, by definition of our heuristic, adding setups to the heuristic solution cannot improve the solution. The second inequality holds because the new solution has at most $n^{*}-1$ additional setups and less holding cost compared to the optimal solution. Furthermore, the last inequality holds because $C^{*} \geq K n^{*}$. In conclusion, we have $\frac{C^{H^{*}}}{C^{*}} \leq 2$.

The following example shows that this bound is tight.

Example 2 Consider a problem instance with $T=2 n(n \in \mathbb{N}), K=h=1, d_{t}=2 \varepsilon$ for $t$ odd and $d_{t}=1-\varepsilon$ for $t$ even. For $\varepsilon$ sufficiently small heuristic $H^{*}$ generates a solution with setups in periods $1,3, \ldots, T-1$ with total cost

$$
C^{H^{*}}=n K+n h(1-\varepsilon)=2 n-n \varepsilon .
$$


However, an alternative solution with setup periods $1,2,4, \ldots, T$ has a total cost of

$$
C^{A}=(n+1) K+2 h \varepsilon(n-1)=(n+1)+2(n-1) \varepsilon .
$$

If $C^{*}$ is the cost of the optimal solution, then the worst case ratio satisfies

$$
\frac{C^{H^{*}}}{C^{*}} \geq \frac{C^{H^{*}}}{C^{A}}=\frac{2 n-n \varepsilon}{(n+1)+2(n-1) \varepsilon} \rightarrow 2 \text { for } \varepsilon=\frac{1}{n} \text { and } n \rightarrow \infty .
$$

Theorem 6 and Example 2 show that $H^{*}$ is a heuristic with worst case ratio 2 . This is the best possible worst case ratio for the class of heuristics in which $H^{*}$ is contained (see Van den Heuvel and Wagelmans (2007)). Note that $H^{*}$ is not contained in the class of heuristics considered by Axsäter (1985) and hence the result cannot be derived from this paper. Theorems 7 and 8 show that $H^{*}$ possesses some other nice properties.

Theorem 7 The number of setup periods generated by $H^{*}$ is at most the number of setup periods in any optimal solution.

Proof Consider some order interval $r, \ldots, s-1$ of an optimal solution. It is sufficient to show that $H^{*}$ will generate at most one setup in this interval. Assume this is not the case and let $v$ and $w$ be two consecutive setup periods of the heuristic solution such that $r \leq v<w<s$. By definition of $H^{*}$ there exists a period $p \in\{v+1, \ldots, w\}$ such that

$$
K+h \sum_{i=v+1}^{p-1}(i-v) d_{i}+K+h \sum_{i=p+1}^{w}(i-p) d_{i}<K+h \sum_{i=v+1}^{w}(i-v) d_{i},
$$

which implies

$$
K+h \sum_{i=r+1}^{p-1}(i-r) d_{i}+K+h \sum_{i=p+1}^{w}(i-p) d_{i}<K+h \sum_{i=r+1}^{w}(i-r) d_{i} .
$$

Now add a setup in period $p$ in the optimal solution. Then the interval $r, \ldots, s-1$ has a cost of

$$
\begin{aligned}
K+h \sum_{i=r+1}^{p-1}(i-r) d_{i}+K+h \sum_{i=p+1}^{s-1}(i-p) d_{i} & = \\
K+h \sum_{i=r+1}^{p-1}(i-r) d_{i}+K+h \sum_{i=p+1}^{w}(i-p) d_{i}+h \sum_{i=w+1}^{s-1}(i-p) d_{i} & < \\
K+h \sum_{i=r+1}^{w}(i-r) d_{i}+h \sum_{i=w+1}^{s-1}(i-p) d_{i} & \leq K+h \sum_{i=r+1}^{s-1}(i-r) d_{i},
\end{aligned}
$$

where the last inequality follows from $p>r$. This means we have found a better solution than the optimal solution, which is a contradiction. 
Theorem 8 Let $r, \ldots, s-1$ be some order interval generated by $H^{*}$. Then an optimal solution with minimum number of setups has at most 2 setups in this interval.

Proof Assume we have an optimal solution with consecutive setups in periods $u<v<w$ with $u \geq r$ and $w \leq s-1$ and minimum number of setups. By definition of optimality we have

$$
(v-u) \sum_{i=v}^{w-1} d_{i}>K .
$$

That is, the additional cost of removing the setup in period $v$ is larger than the setup cost. Note that we have strict inequality because we assume it is an optimal solution with minimum number of setups. Because $r \leq u$

$$
(v-r) \sum_{i=v+1}^{w-1} d_{i} \geq(v-u) \sum_{i=v+1}^{w-1} d_{i}>K .
$$

This means that adding a setup in period $v$ in the solution generated by $H^{*}$ would lead to cost reduction and hence $H^{*}$ should have a setup in period $w-1<s-1$. But this is a contradiction with our initial assumption.

The previous theorems immediately imply the following result.

Corollary 9 Let $n^{*}$ be the (minimum) number of setups in an optimal solution and let $n$ be the number of setups generated by $H^{*}$. Then $n \leq n^{*} \leq 2 n$ or $\frac{1}{2} n^{*} \leq n \leq n^{*}$.

A consequence of Corollary 9 is that heuristic $H^{*}$ will not suffer from generating too many setups compared to the number of setups in an optimal solution. Furthermore, the number of setups is not less than half the number of setups of an optimal solution (with minimum number of setups).

We end this section with some remarks about $H^{*}$. First, heuristic $H^{*}$ can also be adapted for the case of time-varying holding cost by using the interpretation that the heuristic generates order intervals covering as many demand periods as possible such that no additional setup may improve the solution. Similar arguments as used in the proof of Theorem 6 show that this heuristic also has a worst case performance of 2 . Second, heuristic $H^{*}$ can be implemented in a backward way having the same worst case performance. These additional properties are similar to the properties of the heuristic presented in Bitran et al. (1984). This heuristic starts a new order if total holding cost in the current order interval exceeds the setups cost. Finally, heuristic $H^{*}$ can be implemented in linear time since there exists a linear time algorithm for the economic lot-sizing problem with time-invariant costs (see Federgruen and Tzur (1991), Wagelmans et al. (1992) and Aggarwal and Park (1993)). 


\section{Application to worst case analysis}

In this section we show that our result may be useful for worst case analysis. Axsäter (1982) presents a problem instance that is used to show that the well-known Silver-Meal heuristic has an infinite worst case performance. The problem instance has a demand $d_{1}>0$ and $d_{t}=\frac{K}{(t-1) h}$ for $t=2,3 \ldots, T$. As in Axsäter (1982), we construct a solution and compare it to the SilverMeal solution. In our case the solution is based on the holding cost bound derived in Section 2. The Silver-Meal heuristic generates a solution with one setup, resulting in a cost of $C^{S M}=T K$, that is a cost of $K$ per period.

We will now construct a solution for this problem instance based on our holding cost bound. Assume period $p$ is the last setup period of the current order interval. Then Theorem 4 shows that the end of the order interval is bounded by the largest period $t$ that satisfies

$$
h \sum_{i=2}^{t}(i-1) d_{i+p-1}=K \sum_{i=2}^{t} \frac{i-1}{i+p-2} \leq K \sum_{i=1}^{t-1} \frac{1}{i} .
$$

Note that (9) is certainly satisfied when the largest term at the left hand side of (9) is smaller than the smallest term at the right side, i.e., when

$$
\frac{t-1}{t+p-2} \leq \frac{1}{t-1} \Leftrightarrow t^{2}-3 t+3+p \leq 0
$$

The length of the largest order interval for which (10) holds equals

$$
t=\left\lfloor\frac{3}{2}+\sqrt{p-\frac{3}{4}}\right\rfloor .
$$

For example, when $p=1$ we make an order for $t=2$ periods and when $p=7$ we make an order to cover $t=4$ periods.

Using (6) we have that $C_{p, p+t}^{H}$, the cost (both setup and holding cost) in the interval $[p, p+$ $t-1]$ of the heuristic solution, satisfies

$$
C_{p, p+t}^{H} \leq K\left(1+\gamma+\log \left(\frac{3}{2}+\sqrt{p-\frac{3}{4}}\right)\right),
$$

whereas $C_{p, p+t}^{S M}$, the cost generated by the Silver-Meal heuristic in the interval $[p, p+t-1]$, equals

$$
C_{p, p+t}^{S M}=K t=K\left\lfloor\frac{3}{2}+\sqrt{p-\frac{3}{4}}\right\rfloor .
$$

Because

$$
\frac{C_{p, p+t}^{S M}}{C_{p, p+t}^{H}} \geq \frac{\left\lfloor\frac{3}{2}+\sqrt{p-\frac{3}{4}}\right\rfloor}{1+\gamma+\log \left(\frac{3}{2}+\sqrt{p-\frac{3}{4}}\right)} \rightarrow \infty \text { as } p \rightarrow \infty
$$

we have that

$$
\frac{C^{S M}}{C^{*}} \geq \frac{C^{S M}}{C^{H}} \rightarrow \infty \text { for } T \rightarrow \infty
$$


where $C^{*}$ is the optimal cost. So using the solution based on the holding cost bound as a benchmark shows that the Silver-Meal heuristic has an infinite worst case ratio, as was shown by Axsäter (1982).

\section{Conclusion}

In this chapter we presented a new structural property for an optimal solution of the ELS problem with time-invariant cost parameters. In any order interval the total holding cost is bounded from above by a quantity proportional to the setup cost and the logarithm of the number of periods in the order interval. Furthermore, we showed by an example that this bound is tight. This means that in an optimal solution the ratio between the holding cost and setup cost can be arbitrarily large. This is in contrast to the classical EOQ model where setup cost and holding cost are perfectly balanced in an optimal solution.

The property was used to construct a new heuristic. In this heuristic a new order is placed when the holding cost exceeds a particular upper bound. We showed that this heuristic has worst case ratio 2. Furthermore, a solution generated by this heuristic has a nice theoretical property: the number of setups of the heuristic solution is at least half the number of setups generated by the optimal solution and at most the number of setups generated by the optimal solution. Finally, we showed that our result may be useful for worst case analysis.

\section{References}

A. Aggarwal and J. K. Park. Improved algorithms for economic lot-size problems. Operations Research, 14:549-571, 1993.

S. Axsäter. Worst case performance for lot sizing heuristics. European Journal of Operational Research, 9(4):339-343, 1982.

S. Axsäter. Performance bounds for lot sizing heuristics. Management Science, 31:634-640, 1985.

G. R. Bitran, T. L. Magnanti, and H. H. Yanasse. Approximation methods for the uncapacitated lot size problem. Management Science, 30(9):1121-1140, 1984.

A. Federgruen and M. Tzur. A simple forward algorithm to solve general dynamic lot sizing models with $n$ periods in $\mathrm{O}(n \log n)$ or $\mathrm{O}(n)$ time. Management Science, 37:909-925, 1991.

R. L. Graham, D. E. Knuth, and O. Patashnik. Concrete Mathematics: A Foundation for Computer Science. Reading, MA: Addison-Wesley, 2nd edition, 1994. 
E. A. Silver, D. F. Pyke, and R. Petersen. Inventory management and production planning and scheduling. Wiley, New York, 3rd edition, 1998.

W. van den Heuvel and A.P.M. Wagelmans. Worst case analysis for a general class of on-line lot-sizing heuristics. Technical Report EI 2007-46, Erasmus University Rotterdam, 2007.

C. P. M. van Hoesel and A. P. M. Wagelmans. Parametric analysis of setup cost in the economic lot-sizing model without speculative motives. International Journal of Production Economics, $66: 13-22,2000$.

A. P. M. Wagelmans, C. P. M. van Hoesel, and A. Kolen. Economic lot sizing: An $\mathrm{O}(n \log n)$ algorithm that runs in linear time in the Wagner-Whitin case. Operations Research, 40: S145-S156, 1992.

H. M. Wagner and T. M. Whitin. Dynamic version of the economic lot size model. Management Science, 5:89-96, 1958.

U. Wemmerlöv. The ubiquitous EOQ - Its relation to discrete lot sizing heuristics. International Journal of Operations and Production Management, 1(3):161-179, 1981. 